\title{
A FIXED POINT THEOREM FOR MANIFOLDS
}

\author{
JAN W. JAWOROWSKI ${ }^{1}$
}

ABstract. A Lefschetz type fixed point theorem is proved extending a recent theorem by Robert F. Brown. It deals with compact maps of the form $f:(M-U, X) \rightarrow(M, M-U)$, where $M$ is an $n$-manifold, $X$ is an $(n-2)$-connected ANR which is closed in $M$ and $U$ is an unbounded component of $M-U$. The map $f$ defines maps $u: M-U \rightarrow M-U$ and $v: M \rightarrow M$; the Lefschetz numbers of $u$ and $v$ are defined and are shown to be equal; and if this number is nonzero then $f$ has a fixed point.

Robert F. Brown [2] proved a generalization of the Brouwer fixed point theorem by making use of a retraction theorem of Bing [1]. A special case of Bing's retraction theorem was used earlier by Henderson and Livesay [5] to prove a theorem which is now a special case of the Brown theorem.

The purpose of this note is to prove a theorem which extends Brown's result in a similar sense as the Lefschetz fixed point theorem extends the Brouwer fixed point theorem. We use the ideas and results of [6].

Let $V$ be a vector space over a field $K$. An endomorphism $f: V \rightarrow V$ will be said to be of finite type if there exists an integer $m$ such that $f^{m} V$ is finite dimensional. In this case, according to Definition (2.3) of [6], the trace $\operatorname{tr} f \in K$ of $f$ is defined.

If now $V=\left\{V_{n}\right\}$ is a graded vector space and $f: V \rightarrow V$ is an endomorphism of degree zero, then $f$ is said to be a Lefschetz endomorphism if each $f_{n}: V_{n} \rightarrow V_{n}$, is of finite type and all but a finite number of them are zero. In this case the Lefschetz number $\Lambda f$ $=\sum_{n}(-1)^{n} \operatorname{tr} f_{n}$ is defined.

In order to define the Lefschetz number $\Lambda f$ of a map $f: X \rightarrow X$ of a topological space $X$ for the purpose of this paper, we may use any functor $H_{*}$ from the topological category to the category of graded vector spaces (over $K$ ) and homomorphisms of degree zero, such that $H_{*}$ satisfies the homotopy axiom, the dimension axiom, and agrees with the usual homology on the category of compact polyhedra. Thus $H_{*}$ may be the singular homology or the Cech homology, for instance.

Received by the editors December 26, 1969.

AMS 1969 subject classifications. Primary 5485; Secondary 5478, 5560.

Key words and phrases. Manifold, retraction, absolute neighborhood retract, compact map, Lefschetz map, $\Delta$-map, fixed point.

1 This research was supported by NSF Grant GP 8928.

Copyright (c) 1971, American Mathematical Society 
Let $X$ be a topological space, $A \subset X$ and $f: A \rightarrow X$. Then the fixed point set of $f$ is $F_{f}=\{x \in A \mid f x=x\}$. A map $f: X \rightarrow X$ is said to be a Lefschetz map provided that:

$\left(1^{\circ}\right)$ The induced homomorphism $f_{*}=H_{*} f: H_{*} X \rightarrow H_{*} X$ is a Lefschetz endomorphism, in which case the Lefschetz number $\Lambda f$ of $f$ is defined to be $\Lambda f=\Lambda\left(f_{*}\right)$;

$\left(2^{\circ}\right)$ The condition $\Lambda f \neq 0$ implies $F_{f} \neq \varnothing$.

A topological space $X$ is said to be a $\Lambda$-space (see [6, (2.6)]) if every compact map $f: X \rightarrow X$ is a Lefschetz map. It was shown in $[6,(5.2)]$, that ANR's are $\Lambda$-spaces (see also [3]).

The following theorem is the main result of this note:

THEOREM 1. Let $M$ be an n-manifold (with or without boundary), let $X$ be an (n-2)-connected $A N R$ imbedded as a closed subset of $M$ and let $U$ be a component of $M-X$ whose closure is not compact. Let $f:(M-U, X) \rightarrow(M, M-U)$ be a compact map and let $f^{\prime}: X \rightarrow M-U$ denote the map defined by the restriction of $f$. Then there exist Lefschetz maps $u: M-U \rightarrow M-U$ and $v: M \rightarrow M$ such that $u$ is an extension of $f^{\prime}$, $v$ is an extension of $f, \Lambda u=\Lambda v$ and $F_{v} \subset F_{u} \cap F_{f}$.

In particular, if $\Lambda v \neq 0$ then $f$ has a fixed point.

Corollary (Brown [2]). Let $M, X, U$ and f be as in Theorem 1 and assume that $M$ is acyclic. Then $f$ has a fixed point.

For, in this case, the Lefschetz number of $v: M \rightarrow M$ is equal to one.

We shall first prove

THEOREM 2. Suppose that a map $f: X \rightarrow X$ can be factored through a $\Lambda$-space $Y$ :

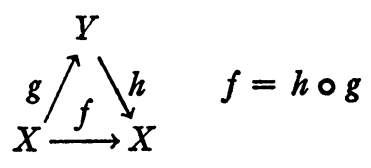

such that: either (a) $g$ is compact; or (b) $Y$ is Hausdorff and $h$ is compact. Then $f$ is a Lefschetz map, $\Lambda f=\Lambda(g \circ h)$ and $h\left(F_{\text {ooh }}\right) \subset F_{f}$.

(Compare [6, (3.1)].)

Proof. Condition (a) or (b) implies that $g \circ h: Y \rightarrow Y$ is compact. Since $Y$ is a $\Lambda$-space, $(g \circ h)_{*}=g_{*} \circ h_{*}: H_{*} Y \rightarrow H_{*} Y$ is a Lefschetz endomorphism; moreover, $\operatorname{tr} f_{*}=\operatorname{tr}\left(h_{*} \circ g_{*}\right)=\operatorname{tr}\left(g_{*} \circ h_{*}\right)$ (see [6, (2.4) ]). It follows that $f_{*}$ is a Lefschetz endomorphism and $\Lambda f_{*}$ $=\Lambda(g \circ h)_{*}$, so $\Lambda f=\Lambda(g \circ h)$. If $y_{0} \in F_{o \circ h}$ then clearly $h y_{0} \in F_{f}$. Thus if $\Lambda f \neq 0$ then $\Lambda(g \circ h) \neq 0$; consequently $F_{g \circ h} \neq 0$ and hence $F_{f} \neq 0$. 
TheOREM 3. A manifold (with or without boundary) is a $\Lambda$-space.

Proof. If $M$ is metrizable then it is a local ANR, and hence an ANR by [4]. Consequently, in this case, it is a $\Lambda$-space by $[6,(5.2)]$. The general case will follow from

LEMмa. Every compact subspace of an $n$-manifold $M$ is contained in a metrizable manifold (with a countable basis).

Proof. Let $K \subset M$ be compact. Then $K$ can be covered by closed $n$-disks $D_{1}, \cdots, D_{s}$ whose interiors $B_{i}=\operatorname{Int} D_{i}, i=1, \cdots, s$, cover $K$. Thus $K$ is metrizable with a countable basis and therefore $N$ $=B_{1} \cup \ldots \cup B_{\mathrm{s}}$ is a manifold with these properties containing $K$.

To prove the general case of Theorem 3, suppose that $f: M \rightarrow M$ is a compact map. Then, by the Lemma, $K=\overrightarrow{f M}$ is contained in a metrizable manifold $N$. Thus we obtain a factorization

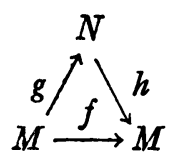

where $g$ is defined by $f$ and $h$ is the inclusion. Since $g$ is compact and $N$ is a $\Lambda$-space, we may apply Theorem 2 to conclude that $f$ is a Lefschetz map and that $\Lambda f=\Lambda(g \circ h)$.

Proof of TheOREM 1. The remaining part of the proof of Theorem 1 is analogous to that of [2]. By Bing's Retraction Theorem [1], there exists a retraction $X \cup U \rightarrow X$ which extends to a retraction $r: M \rightarrow M-U$. We have the factorization

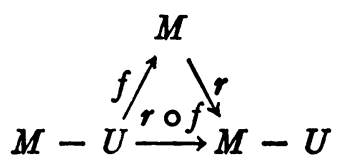

Set $u=r$ of: $M-U \rightarrow M-U, v=f$ or: $M \rightarrow M$. By Theorem 3, $M$ is a $\Lambda$-space; and by Theorem $2, \Lambda u=\Lambda v$. Suppose that $x_{0} \in F_{v}$, i.e., $f r x_{0}=x_{0}$. Then $y_{0}=r x_{0} \in F_{u}$. If we suppose that $x_{0} \in U$, then $y_{0}=r x_{0}$ $\in X$, so $f y_{0}=x_{0} \in M-U$, since $f X \subset M-U$, which is a contradiction. It follows that $x_{0} \in M-U$ and hence $r x_{0}=x_{0}$. Thus $x_{0} \in F_{u}$ and $x_{0} \in F_{f}$.

\section{REFERENCES}

1. R. H. Bing, Retractions onto ANR's, Proc. Amer. Math. Soc. 21 (1969), 618620. MR 39 \#940.

2. Robert F. Brown, $A$ fixed point theorem for open Q-acyclic n-manifolds, Proc. Amer. Math. Soc. 21 (1969), 621-622. MR 39 \#941. 
3. A. Granas, The Hopf-Lefschetz fixed point theorem for non-compact ANR-s, Proc. Sympos. on Infinite Dimensional Topology, Baton Rouge, Louisiana, 1967.

4. O. Hanner, Retraction and extension of mappings of metric and non-metric spaces, Ark. Mat. 2 (1952), 315-360. MR 14, 396; 1278.

5. D. Henderson and G. Livesay, Another generalization of Brouwer's fixed point theorem, Proc. Amer. Math. Soc. 19 (1968), 176-177. MR 36 \#2134.

6. J. W. Jaworowski and M. J. Powers, $\Delta$-spaces and fixed point theorems, Fund. Math. 64 (1969), 157-162. MR 39 \#3476.

INDIANA UNIVERSITy, BLOOMINGTON, INDIANA 47401 